\title{
ubtor Mutation Causes Motor Hyperactivity by Activating mTOR Signaling in Zebrafish
}

\author{
Tiantian Wang ${ }^{1} \cdot$ Mingshan Zhou $^{1} \cdot$ Quan Zhang $^{1} \cdot$ Cuizhen Zhang ${ }^{1} \cdot$ \\ Gang Peng ${ }^{1}$
}

Received: 31 December 2020/Accepted: 8 April 2021/Published online: 26 July 2021

(C) The Author(s) 2021

\begin{abstract}
Mechanistic target of rapamycin (mTOR) signaling governs important physiological and pathological processes key to cellular life. Loss of mTOR negative regulators and subsequent over-activation of mTOR signaling are major causes underlying epileptic encephalopathy. Our previous studies showed that UBTOR/KIAA1024/ MINARI acts as a negative regulator of mTOR signaling, but whether UBTOR plays a role in neurological diseases remains largely unknown. We therefore examined a zebrafish model and found that ubtor disruption caused increased spontaneous embryonic movement and neuronal activity in spinal interneurons, as well as the expected hyperactivation of mTOR signaling in early zebrafish embryos. In addition, mutant ubtor larvae showed increased sensitivity to the convulsant pentylenetetrazol, and both the motor activity and the neuronal activity were up-regulated. These phenotypic abnormalities in zebrafish embryos and larvae were rescued by treatment with the mTORC1 inhibitor rapamycin. Taken together, our findings show that ubtor regulates motor hyperactivity and epilepsy-like behaviors by elevating neuronal activity and activating mTOR signaling.
\end{abstract}

Supplementary Information The online version contains supplementary material available at https://doi.org/10.1007/s12264-02100755-z.

Tiantian Wang and Mingshan Zhou contributed equally to this work.

Gang Peng

gangpeng@fudan.edu.cn

1 State Key Laboratory of Medical Neurobiology, Ministry of Education Frontiers Center for Brain Science, and Institutes of Brain Science, Fudan University, Shanghai 200032, China
Keywords Ubtor · Hyperactivity · Epilepsy · mTOR · Zebrafish

\section{Introduction}

Mechanistic target of rapamycin (mTOR) is an evolutionarily conserved serine-threonine protein kinase. The mTOR signaling plays important roles in various physiological and pathological processes of cellular life $[1,2]$. The lossof-function of negative regulators of mTOR triggers behavioral abnormalities and neurological diseases by over-activating mTOR signaling [3]. For example, absence of the negative regulator tuberous sclerosis complex (TSC) 1 or TSC 2 results in susceptibility to hamartoma formation and epilepsy $[4,5]$. Recently, mutations in the DEP domain containing protein 5 gene (DEPDC5) have been identified as a crucial genetic cause of focal epilepsy [6]. DEPDC5 forms the mTORC1 inhibitory complex GATOR1 with NRPL2 and NRPL3 to block the activation of mTORC1 [7]. In a mouse model, adults with conditional knockout (cKO) of Depdc5 exhibit cortical defects and increased susceptibility to epilepsy [8]. And depdc5 knockdown in the zebrafish model leads to early-onset phenotypic features associated with mTOR-dependent motor and neuronal hyperactivity [9]. Nevertheless, the molecular functions of newly-identified negative regulators of $\mathrm{mTOR}$ in the occurrence and development of neurological diseases remain elusive.

Ubtor is an unannotated gene found in vertebrate species only. The protein encoded by Ubtor is highly conserved in vertebrates [10]. Early studies listed Ubtor as a gene that is down-regulated or mutated in tumor tissues [11, 12]. Our previous study showed that Ubtor is a negative regulator of mTOR signaling, and loss of Ubtor promotes neurite and 
cellular growth [10]. It has also been shown that Ubtor/ MINARl plays roles in angiogenesis and breast cancer [13]. However, the in vivo function of Ubtor remains largely unknown.

In this study, we examined the phenotypic features of ubtor mutant zebrafish.

\section{Materials and Methods}

\section{Zebrafish Maintenance and Ethics Statement}

Experiments were performed on $\mathrm{AB}, \mathrm{Tg}(\mathrm{UAS}$ :EGFP), $\operatorname{Tg}($ ubtor:GAL4FF), and ubtor mutant lines. The AB line was acquired from the University of Oregon Zebrafish Facility; the $\operatorname{Tg}(\mathrm{UAS}$ :EGFP) line was a gift from Dr. $\mathrm{K}$ Kawakami [14]; the $\operatorname{Tg}$ (ubtor:GAL4FF) line was generated by an enhancer trap screen, and the generation of the ubtor mutant line was as previously described [10]. All zebrafish were maintained at $28.5^{\circ} \mathrm{C}$ under $14 \mathrm{~h}$ light/ $10 \mathrm{~h}$ dark cycles. All animal studies were carried out according to the National Research Council (NRC) Guide for the Care and Use of Laboratory Animals and the Fudan University Regulations on Animal Experiments (Approval \#170223-101).

\section{Drug Treatments}

PTZ and rapamycin were from Sigma (St. Louis, USA). PTZ stock was freshly prepared in nuclease-free water. Rapamycin stock was dissolved in dimethyl sulfoxide (DMSO) and stored at $-20^{\circ} \mathrm{C}$. All drugs were diluted in embryonic medium to working concentrations indicated in the text. Zebrafish embryos were exposed to the drugs by soaking and kept in the same solution during behavioral testing.

\section{Motor Activity Analysis}

To assess coiling behavior inside the chorion, 28-h postfertilization (hpf) zebrafish embryos in their chorion were acclimated to room temperature for $30 \mathrm{~min}$, then transferred into a $1.2-\mathrm{mm}$ wide slot in $2.5 \%$ agarose, submerged in embryonic medium, and imaged using a camera (Point Grey, BC, Canada) at $30 \mathrm{~Hz}$. Recorded videos were inspected and the coiling movements of embryos were manually counted.

To assess coiling behavior outside the chorion, embryos at $10 \mathrm{hpf}$ were gently dechorionated using tweezers. A custom-made $5 \times 4$ grid was laser-cut into a $70 \mathrm{~mm} \times 50$ $\mathrm{mm} \times 2 \mathrm{~mm}$ (length $\times$ width $\times$ thickness) acrylic plate to hold the embryos. A single 28-hpf zebrafish was placed in each grid well, and the coiling behaviors were recorded at $30 \mathrm{~Hz}$.

To assess PTZ-induced motor activity, zebrafish larvae at $4 \mathrm{dpf}$ were placed in a 24 -well plate, with a single zebrafish and $1 \mathrm{~mL}$ embryonic medium in each well. Before PTZ induction, all larvae were adapted to the new environment for $10 \mathrm{~min}$. PTZ stock was added to each well and the larval movements were recorded immediately for $10 \mathrm{~min}$. The recorded videos were processed and analyzed with custom-written MATLAB scripts.

\section{In situ Hybridization (ISH)}

Whole-mount in situ hybridization of zebrafish embryos or dissected brain tissue was performed as described previously [15]. Digoxigenin-labeled antisense RNA probes were detected with the alkaline phosphatase-conjugated digoxigenin antibody Fab fragment (1:5000, Roche, Basel, Switzerland) and the alkaline phosphatase substrate NBT/ BCIP (1:50, Roche). Probe constructs were generated by RT-PCR using the primers listed in Table 1, followed by standard DNA cloning methods.

\section{Calcium Imaging}

Zebrafish embryos at $28 \mathrm{hpf}$ were mounted in $1.8 \%$ lowmelting agarose and the agarose around the tail was carefully removed. Images were acquired at $20 \mathrm{~Hz}$ using a spinning disk microscope (Andor, Belfast, Northern Ireland). The recorded images were processed with the NoRMCorre and CaImAn packages to extract neuronal $\mathrm{Ca}^{2+}$ signals $[16,17]$. Then, the MLspike method [18] was applied to extract spiking activity from the $\mathrm{Ca}^{2+}$ signals.

Table 1 Primers used to generate probe constructs used in this study.

\begin{tabular}{ll}
\hline Probe & Sequence \\
\hline ubtor-forward & 5'-CTTCCCAAAATCAACAATCAAAACAAC \\
ubtor-reverse & 5'-GAACACCTGCTCCACTAGGTAG \\
c-fos-forward & 5'-CCCGAGCTCTTACCCCAAAA \\
c-fos-reverse & 5'-GCAATGGTTAGGCAGCACAC \\
vglut2a-forward & 5'-AGGGAGCCTGCTGGTTTTAG \\
vglut2a-reverse & 5'-CGACAGCCAACACTAGAAATGA \\
evx2-forward & 5'-TTTGGGAACCACCACAACGA \\
evx2-reverse & 5'-CTGGGCGTACACTGGAATGA \\
slc6a5-forward & 5'-CAAGAAAATGTCATGCGAAGGGTA \\
slc6a5-reverse & 5'-CCACATACGGGAATGTTGCTG \\
pax 2 -forward & 5'-TAACACGCAAATGGACGGGA \\
pax $2 b$-reverse & 5'-CGCTGGACGATAAACTGGGT \\
gad2-forward & 5'-TCCAGTCTTCGTGCTGTTGG \\
gad2-reverse & 5'-AGGGACACGGGAGTAACAGG \\
\hline
\end{tabular}




\section{Total RNA Extraction and Reverse Transcription}

The zebrafish embryos were placed in an ice-water bath, anaesthetized, and transferred into a 1.5-mL Eppendorf tube. $300 \mu \mathrm{L}$ of lysis buffer containing $\beta$-mercaptoethanol was added and the embryos were ground using a plastic pestle (Sangon Biotech, Shanghai, China). Total RNA extraction from the spinal tissue included the muscle tissue surrounding the spinal cord. The RNA of the samples was extracted using a total-RNA extraction kit (Sangon Biotech). The reverse transcription reactions were conducted using a PrimeScript RT reagent kit (TaKaRa, Shiga, Japan).

\section{Quantitative Real-Time PCR (qRT-PCR)}

qRT-PCR was performed as previously described [15]. The results were normalized to $\beta$-actin and calculated by the normalized $C_{\mathrm{T}}$ values $\left(2^{-\Delta \Delta \mathrm{Ct}}\right)$. The primers used in $\mathrm{qRT}$ PCR assays are listed in Table 2.

\section{Western Blot}

Zebrafish tissues were lysed in $1 \times$ SDS sample buffer (50 $\mathrm{mmol} / \mathrm{L}$ Tris- $\mathrm{HCl} \mathrm{pH} 6.8,2 \% \mathrm{SDS}, 0.01 \%$ bromophenol blue, $10 \%$ glycerol, and $100 \mathrm{mmol} / \mathrm{L}$ dithiothreitol) and boiled at $98{ }^{\circ} \mathrm{C}$ for $10 \mathrm{~min}$. Proteins were resolved by $10 \%$ SDS-PAGE and transferred onto nitrocellulose membranes, which were incubated overnight with primary antibodies (anti-p-S6, Cell Signaling Technology, Boston, USA, cat\#4858S; anti-RPS6, Proteintech, Chicago, USA, cat\#14823-1-AP; and anti- $\beta$-actin, Proteintech, cat\#66009-1-Ig), washed three times, and incubated with Horseradish Peroxidase (HRP)-conjugated secondary antibodies (anti-mouse IgG, Cell Signaling Technology,

Table 2 Primers used in qRT-PCR assays.

\begin{tabular}{|c|c|}
\hline Primer & Sequence \\
\hline$\beta$-actin-forward & 5'-GCTAGTGCGGAATATCATCTGC \\
\hline$\beta$-actin-reverse & 5'-TCCCATACCAACCATGACACC \\
\hline$c$-fos-forward & 5'-AGCAGACGAGCAAGGAAATACAAGAC \\
\hline$c$-fos-reverse & 5'-ATGAAGAGATCGCCGTGACAGTTG \\
\hline vglut $2 a$-forward & 5'-CAAGACTGGCGGCAAACAGGG \\
\hline vglut $2 a$-reverse & 5'-GACAGCCCCAGCATAGGAACC \\
\hline evx2-forward & 5'-CACCACAACGACAACACTTCC \\
\hline$e v x 2$-reverse & 5'-GAATTGCTGTCCGAGAAACCTTTA \\
\hline slc6a5-forward & 5'-ACCTATGTCAGCCCTAGCGA \\
\hline slc6a5-reverse & 5'-GACCAACACCACATACGGGA \\
\hline $\operatorname{pax} 2 b$-forward & 5'-CAGGCGAGTGAATACAGCCT \\
\hline pax $2 b$-reverse & 5'-TTCGCTCCCAGGAACCATTC \\
\hline gad2-forward & 5'-GCGCTACCTGGAGGACAAAG \\
\hline gad2-reverse & 5'-CAGGTTTTGGTGCGGTAAGT \\
\hline
\end{tabular}

cat\#7076S; and anti-rabbit IgG, Cell Signaling Technology,cat\#7074S). Immuno-signals were generated by chemiluminescence (Cell Signaling Technology, cat\#12757), scanned by a FluorChem E system (ProteinSimple, Silicon Valley, USA), and analyzed using ImageJ software.

\section{Immunofluorescence Analysis}

The immunofluorescence analysis was carried out as previously described ${ }^{[10]}$. In brief, zebrafish were fixed with $4 \%$ paraformaldehyde (PFA) for $2 \mathrm{~h}$ at room temperature. The fixed embryos were washed with PBS and then post-fixed with acetone at $-20{ }^{\circ} \mathrm{C}$ for $20 \mathrm{~min}$, blocked, and incubated overnight with primary antibodies at $-4{ }^{\circ} \mathrm{C}$. The embryos were then washed and incubated with secondary antibodies.

\section{Statistical Analysis}

Statistical analysis was performed with GraphPad Prism 7. Data are shown as the mean \pm SEM. Student's $t$-tests were conducted when appropriate. $P<0.05$ was considered statistically significant: $* P<0.05, * * P<0.01$, and $* * * P<0.001$.

\section{Results}

\section{ubtor is Expressed in Spinal Interneurons in Ze- brafish Embryos}

We performed in situ hybridization (ISH) analysis of 28-hpf zebrafish embryos and found that ubtor mRNA was specifically enriched in the spinal cord and hindbrain, with little to no signal detected in other structures (Fig. 1A). To determine the timeline of ubtor expression in the spinal cord during development, we analyzed the presence of ubtor transcripts by ISH at several stages of embryonic development: 24, 28, and $32 \mathrm{hpf}$. Previous studies have shown that zebrafish and mammalian spinal cord neurons have a similar distribution, and different types of neurons are stereotypically arranged along the dorsoventral axis [19] (Fig. S1A). We found that the ubtor gene was already specifically expressed in interneurons along the dorsoventral axis in the spinal cord of embryos at $24 \mathrm{hpf}$, but not on the ventral side of the cord (Fig. 1B). EGFP expression patterns in the transgenic line $\mathrm{Tg}$ (ubtor:Gal4;UAS:EGFP) were similar to the results of ubtor ISH in the spinal cord (Fig. 1C and S1). Meanwhile, the expression pattern of ubtor in spinal interneurons consistently increased with time over the course of development. To further examine the distribution of ubtor-expressing interneurons in the 
spinal cord, we carried out double-labeling by ubtor:GFP and neural markers (slc6a5/glyT2 and gad2) by using immunofluorescence and ISH assays. Extensive overlap of ubtor:GFP and slc6a5/glyT2 was observed, and some overlap of ubtor:GFP and gad 2 was seen in the spinal cord of the wild-type at $28 \mathrm{hpf}$ (Fig. S1B, C). These results indicate that the neurons expressing ubtor are mainly located in the middle of the spinal cord and ubtor may play a role in spinal interneuron functions.

A
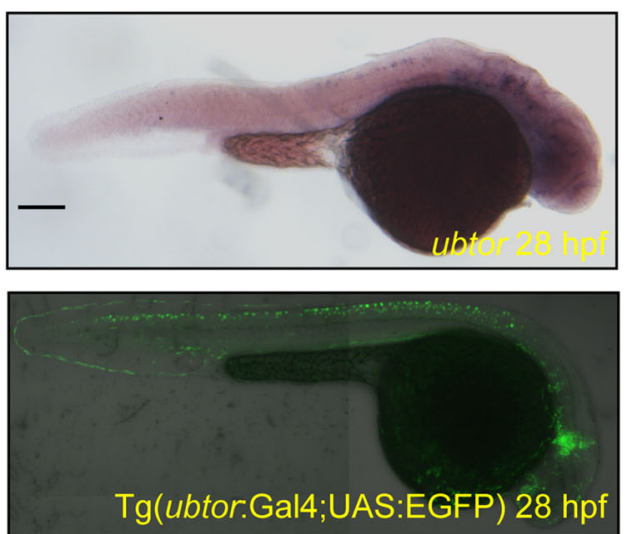

\section{ubtor Mutants Show Hyperactive Spontaneous Movement}

Spinal interneurons have been identified in the regulation of movement behaviors and are key to connection and integration in the spinal circuits of zebrafish [20]. Zebrafish embryos exhibit early stereotyped motor activity starting at $17 \mathrm{hpf}$, showing repetitive twitches (tail flicks) and coils (complete rotations) at regular intervals, the frequencies varying with age [21]. Spontaneous movement frequency is controlled by a synchronized, bilateral spinal circuit [22]. Because ubtor is expressed in spinal interneurons at early developmental stages, ubtor may participate in the spinal

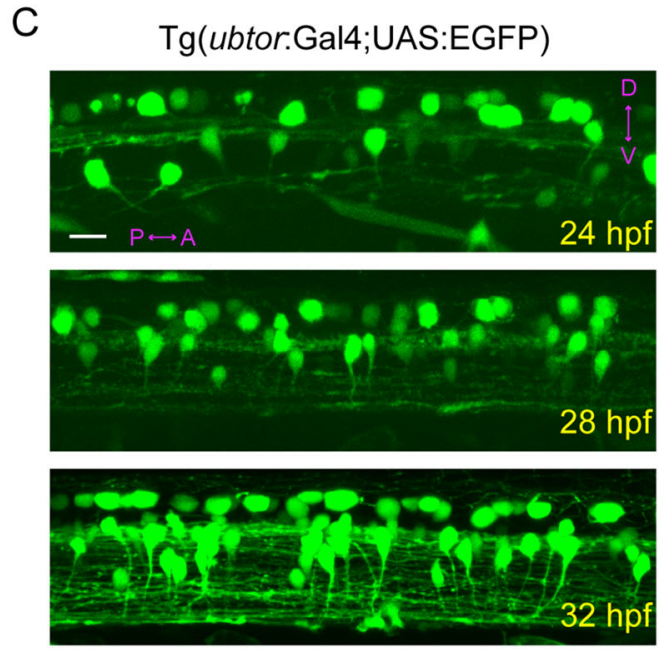

Lateral view

B
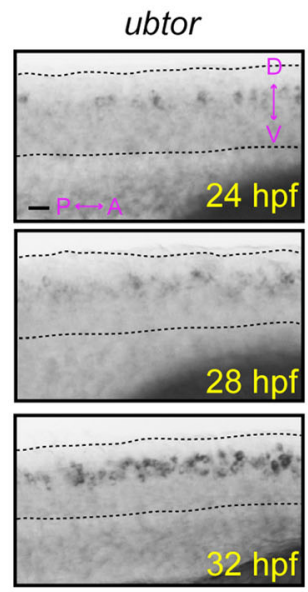

Lateral view

Fig. 1 ubtor is expressed in spinal interneurons and ubtor mutants show increased spontaneous coiling. A ubtor expression (upper) and EGFP expression (lower) in 28-hpf $\mathrm{Tg}$ (ubtor:GAL4;UAS:EGFP) zebrafish embryos (scale bar, $250 \mu \mathrm{m}$ ). B Expression and distribution of the ubtor gene in zebrafish embryo spinal cord tissues at 24, 28, and $32 \mathrm{hpf}$ (dotted lines, spinal cord tissue from hindbrain to somite 20. Scale bar, $60 \mu \mathrm{m}$. C EGFP expression in the spinal cord tissue of embryos at somites $4-10$ of the $\operatorname{Tg}$ (ubtor:GAL4;UAS:EGFP) line
Inside the chorion

E

Outside the chorion
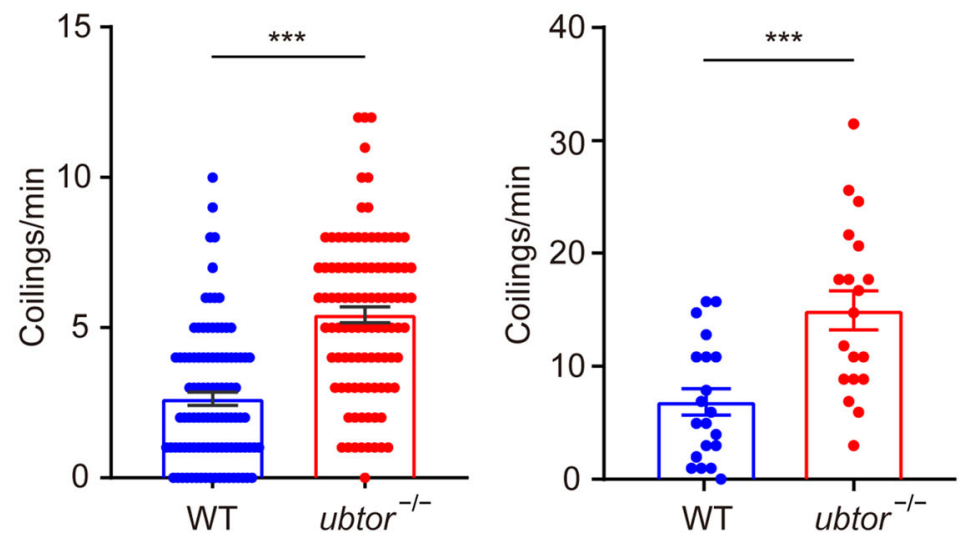

(scale bar, $20 \mu \mathrm{m}$ ). D Frequency of coiling movements of embryos inside the chorion of 28-hpf ubtor mutants and the wild-type (results from five biological repeats, $\mathrm{N}_{\mathrm{WT}}=\mathrm{N}_{u b t o r}{ }^{-1-}=126, t_{198}=8.097$ ). E Frequency of coiling movements outside the chorion of 28-hpf ubtor mutants and the wild-type (two biological repeats, $\mathrm{N}_{\mathrm{WT}}=$ $\left.\mathrm{N}_{\text {ubtor }}{ }^{-1-}=20, t_{37}=3.914\right)$. Values are represented as the mean \pm SEM in $\mathbf{D}$ and $\mathbf{E}$. $* * * P<0.001 ; \mathrm{P}$, posterior; A, anterior; D, dorsal; V, ventral. 
interneuron-related movement. To test this hypothesis, we examined the spontaneous coiling behavior in ubtor mutant embryos. Our results showed that there was no significant difference of coiling behavior in 21-hpf wild-type and ubtor mutant embryos (ubtor mutants: $10.38 \pm 1.307$ coils/ min $v s$ control: $9.875 \pm 1.121$ coils $/ \mathrm{min}, P=0.774$, Fig. S2). Importantly, ubtor mutant embryos exhibited a hyperactive phenotype inside the chorion at $28 \mathrm{hpf}$ (ubtor mutants: $5.43 \pm 0.265$ coils $/$ min $v s$ control: $2.63 \pm 0.222$ coils/min, $P<0.001$, Fig. 1D). A similar phenomenon was present in ubtor mutant embryos outside the chorion at 28 hpf (ubtor mutants: $15.21 \pm 1.763$ coils/min vs control: $6.95 \pm 1.195$ coils $/ \mathrm{min}, P<0.001$, Fig. 1E). Taken together, these data show that ubtor is expressed in spinal interneurons and that its loss-of-function causes hyperactive spontaneous movement in zebrafish embryos.

\section{ubtor Mutation Increases Activity in Spinal Interneurons Without Affecting their Development}

The cell bodies of spinal interneurons (comprising GABAergic, glycinergic, and glutamatergic neurons, Fig. 2A, modified from [20]) are located in the central nervous system and receive signals from sensory (afferent) neurons, which are then transmitted to the motor (efferent) neurons and generate corresponding motor behaviors. To determine the types of interneurons in the spinal cord that express the ubtor gene, we used a mosaic single-cell labeling method. A mixture of a Tol2-UAS:tdTomato plasmid and Tol2-transposase mRNA was microinjected into one-cell stage $\operatorname{Tg}$ (ubtor:Gal4; UAS:EGFP) embryos to sparsely label spinal neurons with the red tdTomato signal. The results showed that tdTomato successfully labeled CiD (circumferential descending), CoPA (commissural primary ascending), and CoSA (commissural secondary ascending) glutamatergic neurons and CoLA (commissural longitudinal), $\mathrm{CoB}$ (commissural bifurcating), and CoSA glycinergic neurons. These labeled glutamatergic and glycinergic neurons were co-labeled with EGFP in transgenic fish, indicating that the neurons expressing ubtor are glutamatergic or glycinergic (Figs S2B and S3).

To determine if ubtor mutation alters the development of spinal neurons, we examined the expression profiles of known interneuron markers using in situ hybridization. Compared with wild-type controls, there were no significant differences in the expression and distribution patterns of the vglut $2 a$ (glutamatergic marker), slc6a5, pax $2 b$ (glycinergic markers), and gad2 (GABAergic marker) genes in the spinal cord of the ubtor mutant (Fig. 2C-E). These results suggest that the ubtor gene mutation does not significantly affect the development of spinal interneurons in zebrafish.
Studies have shown that abnormal neuronal activity is one of the main causes of epilepsy. $C$-fos is an immediateearly gene that is widely used as a molecular marker of neuronal activity $[23,24]$. It is transiently induced in the central nervous system during seizures [25]. To determine whether the hyperactive phenotypic features of ubtor mutant larvae were correlated with increased neuronal activity, we assessed the expression of the $c$-fos gene in the spinal cord neurons of 28-hpf embryos. Using the qRTPCR and ISH methods, we found significantly increased $c$ fos expression levels in spinal interneurons in ubtor mutant larvae compared with their wild-type siblings (Fig. 2F, G). Meanwhile, the number of spinal interneurons expressing $c$-fos was also significantly increased (Fig. $2 \mathrm{H}, \mathrm{I}$ ).

We further investigated the spinal neuron activity by $\mathrm{Ca}^{2+}$ imaging. Because early zebrafish embryos are transparent, $\mathrm{Ca}^{2+}$ imaging has been used in these models to study neural activity, including several models of epilepsy [26, 27]. We bred a $\operatorname{Tg}$ (ubtor:Gal4;UAS:GCaMP5HS) transgenic tool line into the ubtor mutant and found prominent changes in the $\mathrm{Ca}^{2+}$ signals in CiD-type spinal interneurons both in the ubtor mutant and the wildtype (Fig. 3A, B). Importantly, the frequency of burst and spike activity in the $\mathrm{CiD}$ interneurons was higher in ubtor mutant embryos, as inferred from MLSpike analysis [18] (Fig. 3C-E). This suggested that the activity of CiD neurons was higher in the ubtor-mutated group than in the wild-type control group, consistent with up-regulation of the $c$-fos gene in spinal neurons with ubtor depletion. Collectively, these data demonstrate that the loss-offunction of ubtor enhances the activity of spinal interneurons and up-regulates early spontaneous movement in zebrafish.

\section{Spinal Interneurons Exhibit mTORC1 Hyperacti- vation in ubtor Mutant Embryos}

It has been reported that up-regulation of the mTOR signaling pathway affects motor behaviors and neuronal excitability [28]. Our previous studies showed that, in both mammalian cells and zebrafish tissues, depletion of Ubtor up-regulates activity in the mTORC1 pathway [10]. Therefore, we speculated that the increase in spontaneous movement and up-regulated activity of spinal interneurons in 28-hpf zebrafish may be regulated by the mTORC1 pathway. We assessed the phosphorylation state of RPS6, a downstream substrate of mTORC1, by immunofluorescence in ubtor mutants in the $\operatorname{Tg}$ (ubtor:Gal4;UAS:EGFP) background. In this background, EGFP fluorescence represents the expression of the endogenous ubtor gene, $\mathrm{p}-\mathrm{S} 6$ (phosphorylated RPS6 protein) represents the activation status of the mTORC1 pathway, and EGFP neurons colabeled with p-S6 represent the activation of the mTORC1 
A

Dorsal

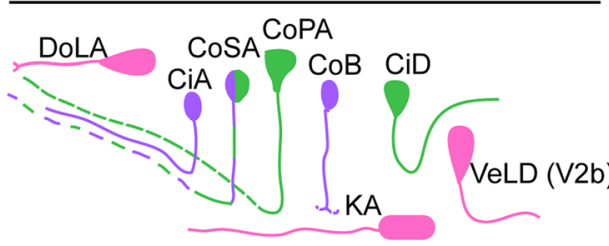

Ventral

Inhibitory

Neurotransmitters

GABA Glycine Glutamate
Excitatory

Neurotransmitters
B

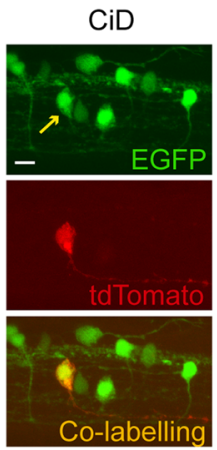

CoPA

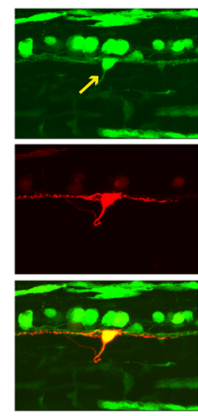

$\cos A$

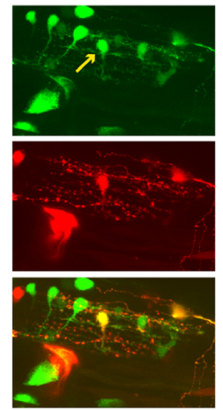

C

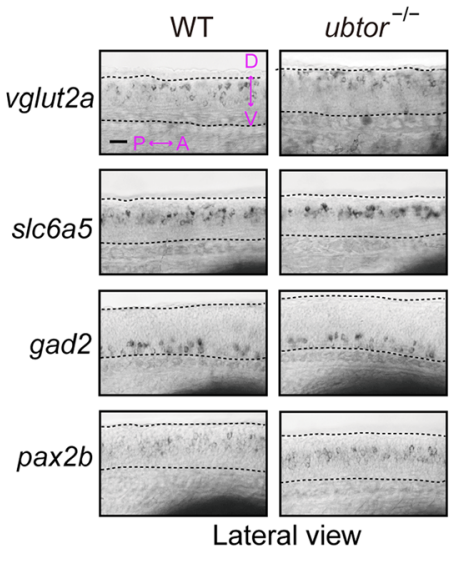

\section{$\mathrm{D}$}
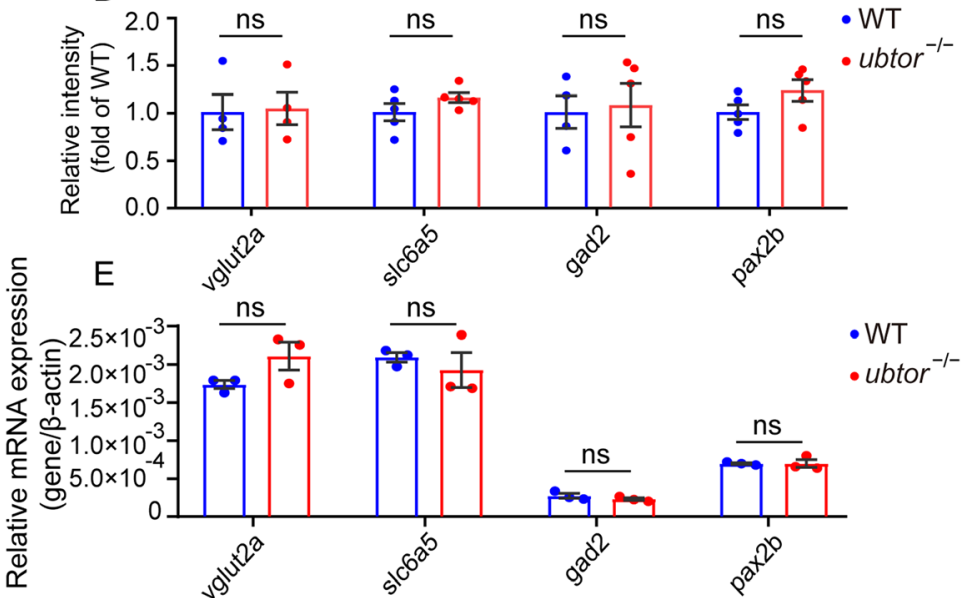

$\mathrm{F}$

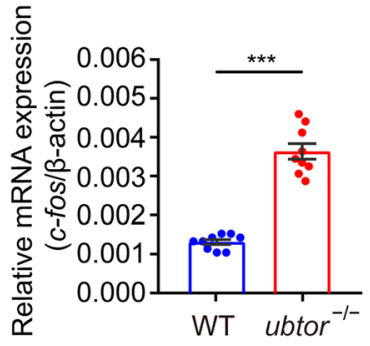

G

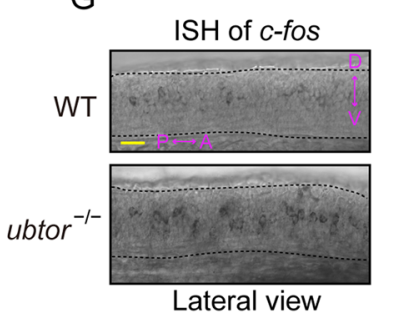

$\mathrm{H}$

I
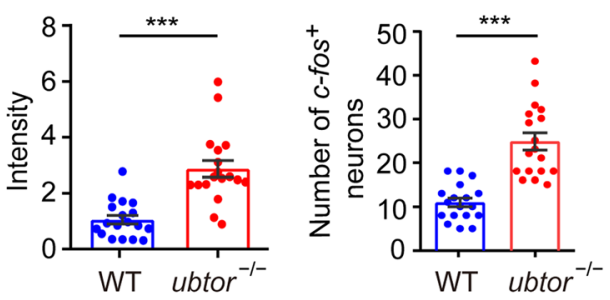

Fig. 2 ubtor mutants show increased activity in spinal interneurons. A Schematic of different types of spinal interneurons (modified from [20]). B Mosaic labeling of spinal interneurons by tdTomato in 28-hpf $\operatorname{Tg}$ (ubtor:GAL4;UAS:EGFP) transgenic embryos (scale bar, $20 \mu \mathrm{m}$ ). Arrows indicate mosaic labelling interneurons. C Expression and distribution of spinal interneuron markers in spinal somites 4-10 (scale bar, $60 \mu \mathrm{m}$ ). D Quantification of relative intensity of spinal interneuron markers (vglut $2 a: \mathrm{N}_{\mathrm{WT}}=\mathrm{N}_{\text {ubtor }}{ }^{-1-}=5, t_{6}=0.150$; slc6a5: $\mathrm{N}_{\mathrm{WT}}=\mathrm{N}_{\text {ubtor }}{ }^{-1-}=5, t_{8}=1.462 ;$ gad2: $\mathrm{N}_{\mathrm{WT}}=\mathrm{N}_{\text {ubtor }}{ }^{-1-}=5, t_{7}$ $=0.2453$; pax $2 b: \mathrm{N}_{\mathrm{WT}}=\mathrm{N}_{\text {ubtor }}{ }^{-1-}=5, t_{8}=1.662$ ). E RT-qPCR analyses of expression levels of spinal interneuron markers in the trunk tissues of 28-hpf embryos (three biological repeats, each marker: $\mathrm{N}_{\mathrm{WT}}=\mathrm{N}_{\text {ubtor }}{ }^{-1-}=75$ ). F RT-qPCR analysis of $c$-fos

pathway in ubtor neurons. These results showed that, compared with the wild-type control, the number of p-S6positive spinal interneurons in the ubtor mutant increased significantly at $28 \mathrm{hpf}$ (Fig. 4A, B), as did the number of neurons co-labeled with EGFP and p-S6 (Fig. 4C). We further found that the distribution of p-S6-positive neurons expression levels in the trunk tissues of 28-hpf embryos (three biological repeats, $\mathrm{N}_{\mathrm{WT}}=\mathrm{N}_{u b t o r}{ }^{-1-}=75, t_{16}=10.99$ ). G Expression and distribution of $c$-fos in spinal somites 4-10 (scale bar, $40 \mu \mathrm{m}$ ). $\mathbf{H}$ Intensity of $c$-fos-labeled neurons in spinal cord (two biological repeats, $\left.\mathrm{N}_{\mathrm{WT}}=\mathrm{N}_{\text {ubtor }}{ }^{-l-}=18, t_{34}=5.348\right)$. I Numbers of $c$-fos labeled neurons in spinal cord (two biological repeats, $\mathrm{N}_{\mathrm{WT}}=$ $\left.\mathrm{N}_{\text {ubtor }}{ }^{-1-}=18, t_{34}=6.324\right)$. $\beta$-actin served as the internal control. Values are represented as the mean \pm SEM. In D-F, H, and I, *** $P<0.001$. Abbreviations: DoLA, dorsal longitudinal ascending; $\mathrm{CiA}$, circumferential ascending; CoSA, commissural secondary; CoPA, commissural primary ascending; $\mathrm{CoB}$, commissural bifurcating; KA, Kolmer-Agduhr; CiD, circumferential descending; VeLD, ventral longitudinal descending.

was similar in the controls and the ubtor mutants (Fig. S4A). Second, when we compared the number of p-S6-positive and EGFP-negative neurons between the control and the ubtor mutants, we found no significant difference (Fig. S4B). These results suggest that disruption 
A
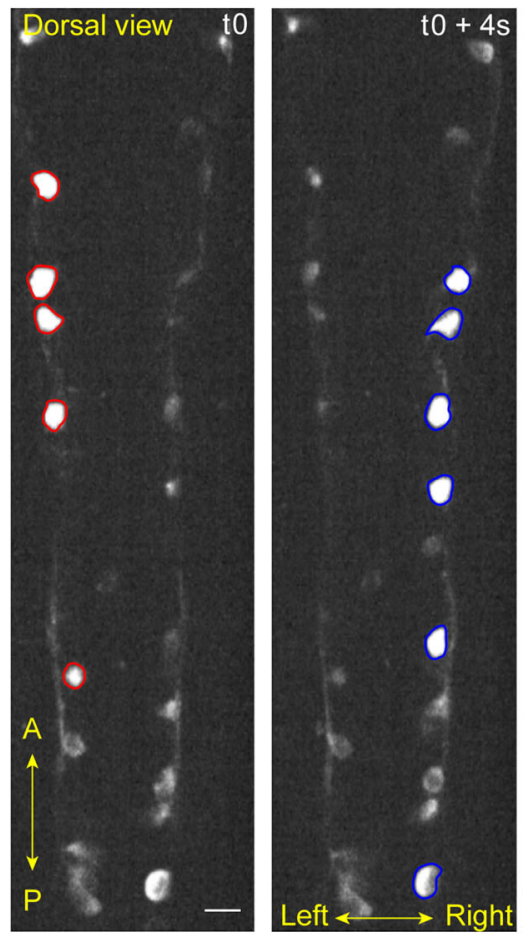

B
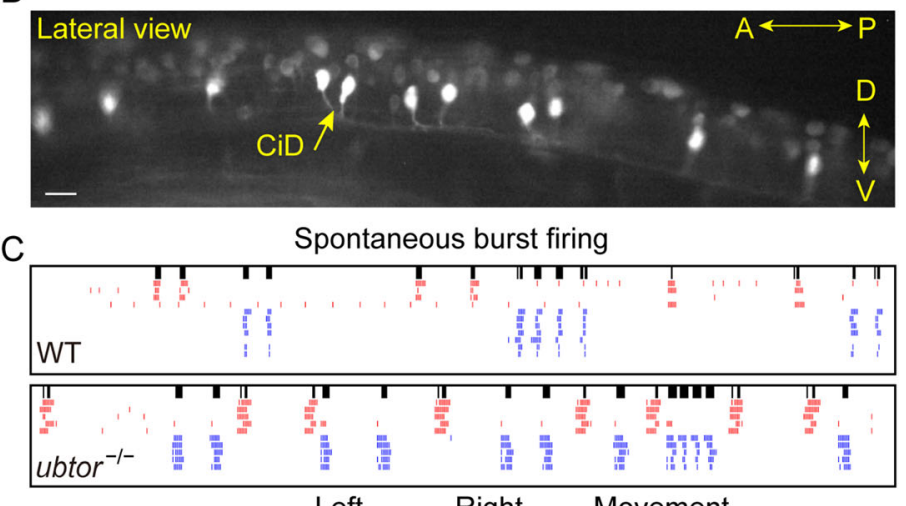

D

$\mathrm{E}$
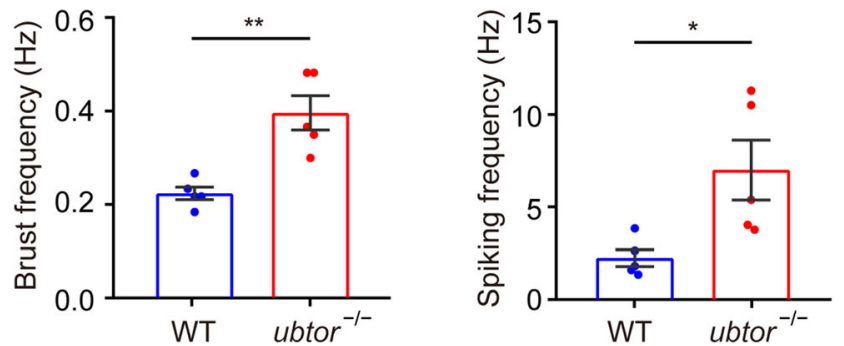

C Rasters of correlated muscle movements and neuronal bursts (black bars, muscle movements; red and blue lines, left and right interneuron spikes estimated by the MLspike method). D, E Quantitative analysis of neuronal burst frequency $\left(\mathrm{N}_{\mathrm{WT}}=\mathrm{N}_{\mathrm{ubtor}}{ }^{-1-}=5, t_{8}=\right.$ 4.396; D and spike frequency $\left(\mathrm{N}_{\mathrm{WT}}=\mathrm{N}_{\text {ubtor }}{ }^{-l-}=5, t_{8}=2.821\right.$; $\mathbf{E}$ of spinal interneurons. Values are represented as the mean \pm SEM in D and E. $* P<0.05, * * P<0.01$.

enhanced activity of spinal interneurons. Studies have shown that the mTORC1 pathway is involved in regulating the excitability of neurons [30]. In order to assess whether the mTORC1 pathway increases early spontaneous movement by regulating the neuronal activity of spinal interneurons in zebrafish, we examined the effect of rapamycin treatment on the activity of spinal neurons. The results showed that rapamycin treatment significantly decreased the c-fos mRNA levels in the spinal interneurons of the 28-hpf ubtor mutants (Fig. 5D). An ISH experiment showed that rapamycin reduced the intensity of expression and number of $c$-fos-positive spinal interneurons in ubtor mutants (Fig. 5E-G). These results suggested that rapamycin inhibits the abnormally up-regulated activity of spinal interneurons in ubtor-mutated zebrafish, but has no detectable effect on the wild-type control group. They also suggest that the activation of mTORC1 pathway activity in ubtor mutants results in the up-regulated activity of spinal interneurons. Taken together, these studies indicate that the depletion of ubtor regulates the activity of the mTORC1 signaling pathway, thereby affecting the activity of neurons, and ultimately controlling the spontaneous movement of zebrafish embryos. 
A

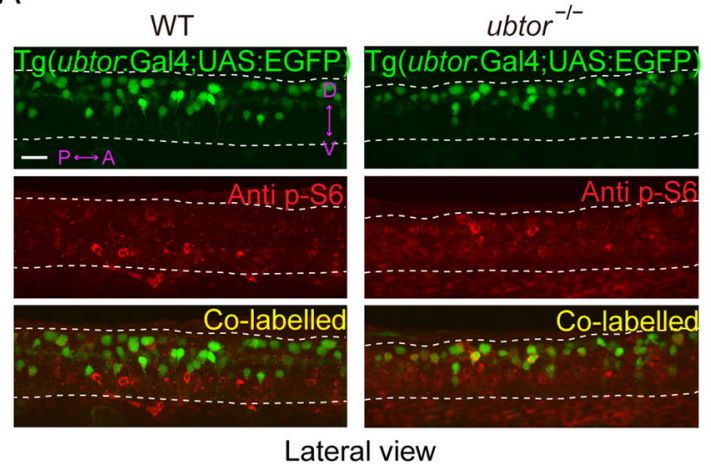

Fig. 4 mTORC1 hyperactivation in the spinal interneurons of ubtor mutant embryos. A p-S6 staining and EGFP signals in 28-hpf embryos (dotted lines outline spinal somites 4-10; scale bar, $40 \mu \mathrm{m}$ ). B Numbers of p-S6-labelled neurons in the spinal cord of 28-hpf embryos (three biological repeats, $\mathrm{N}_{\mathrm{WT}}=22, \mathrm{~N}_{\text {ubtor }}{ }^{-1-}=19, t_{39}=$

\section{The Ubtor Mutant Shows Increased Sensitivity to PTZ}

The results above showed that ubtor depletion induces abnormal motor behaviors and neuronal activity, suggesting that ubtor mutations are involved in epilepsy-like behaviors in zebrafish. PTZ is a GABA receptor inhibitor that increases neuronal activity through the inhibition of GABA signaling networks. PTZ is also a common inducer in kindling models in mice, juvenile models, and adult zebrafish models of epilepsy [25, 31]. We therefore used PTZ to induce epilepsy-like behavior in ubtor mutant zebrafish and wild-type controls at $4 \mathrm{dpf}$. Prior to the PTZ treatment, both control and ubtor mutant 4-dpf larvae had low activity levels (ubtor mutant: $42.77 \pm 17.68 \mathrm{~mm} / \mathrm{min}$ vs control: $21.28 \pm 13.21 \mathrm{~mm} / \mathrm{min}, P=0.3336$, Fig. 6 A, B). After PTZ treatment, both the wild-type and ubtor mutant had significantly increased activity levels. Strikingly, the motor activity of the ubtor mutant was significantly higher than that of the wild-type control (ubtor mutant: $277.6 \pm 33.65 \mathrm{~mm} / \mathrm{min} v s$ control: $134.5 \pm 16.88$ $\mathrm{mm} / \mathrm{min}, P<0.001$, Fig. 6 A, B). To assess mTORC1 activation after PTZ induction, we used a p-S6 Western blot. Before PTZ treatment, both the wild-type and ubtor mutant at 4 dpf had low levels of p-S6 protein. After PTZ induction, the p-S6 protein levels were up-regulated both in the wild-type and ubtor mutant, with a stronger upregulation in the mutant (Fig. 6C). A similar phenomenon was found with regard to $c$-fos expression in the brain and spinal tissues (the latter included muscle tissue surrounding the spinal cord) in response to PTZ treatment in the wildtype and ubtor mutant at 4 and $14 \mathrm{dpf}$ (Fig. 6D, E and S5). These results indicated that the ubtor mutant zebrafish are more prone to motor hyperactivity after treatment with
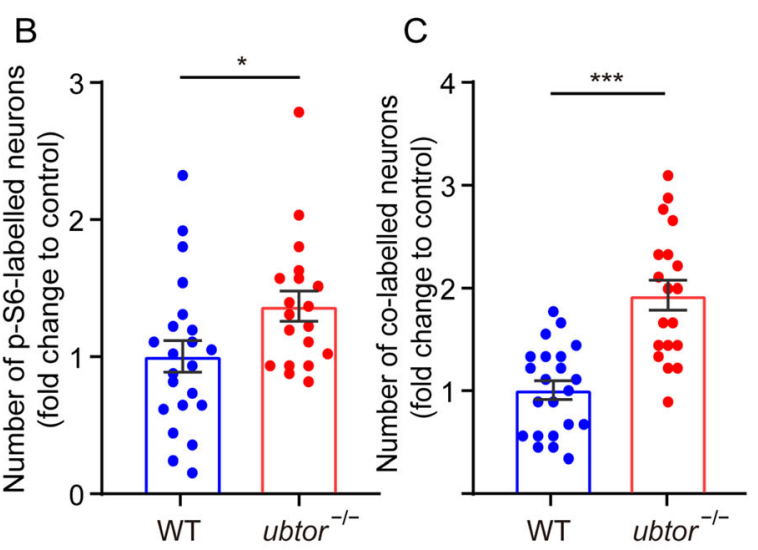

5.538). C Numbers of co-labeled p-S6/EGFP neurons in the spinal cord of 28-hpf embryos (three biological repeats, $\mathrm{N}_{\mathrm{WT}}=22, \mathrm{~N}_{\text {ubtor }}{ }^{-1-}$ $\left.=19, t_{39}=2.265\right)$. Values are represented as the mean $\pm \mathrm{SEM}$ in B and C. $* P<0.05, * * * P<0.001$.

PTZ, along with activation of the mTOR signaling pathway and higher neuronal activity in the brain and spinal cord.

\section{Rapamycin Rescues Seizure Susceptibility Through PTZ-Induction}

Studies have shown that rapamycin inhibits the occurrence and development of seizures and reduces their frequency in several models of epilepsy [32]. Therefore, we next examined whether rapamycin could rescue the seizure susceptibility induced by PTZ exposure in ubtor mutants. At $4 \mathrm{dpf}$, the subject group (treated with rapamycin at 3 dpf) and the control group were exposed to PTZ and their motor behaviors were recorded (Fig. 7A, B). The results showed that rapamycin treatment significantly rescued the amount of movement by ubtor mutants after PTZ exposure, reaching levels similar to those of the wild-type control (ubtor mutant: $135.4 \pm 36.11 \mathrm{~mm} / \mathrm{min} v s$ control: $116.6 \pm$ $36.95 \mathrm{~mm} / \mathrm{min}, P=0.717$, Fig. 7A, B). After the behavioral experiments, we extracted the total protein of 4-dpf larvae and analyzed the p-S6 protein levels to determine the activity of the mTORC1 signaling pathway. Compared with the DMSO control group, rapamycin treatment significantly decreased the p-S6 protein levels both in wild-type and ubtor-mutated zebrafish (Fig. 7C). These results suggested that rapamycin inhibits the PTZ-induced mTORC1 pathway activity in ubtor mutants. ISH and RTqPCR experiments also showed that rapamycin pretreatment rescued the up-regulated expression of $c$-fos in ubtor mutants after PTZ exposure (Fig. 7D, E). 
A

B
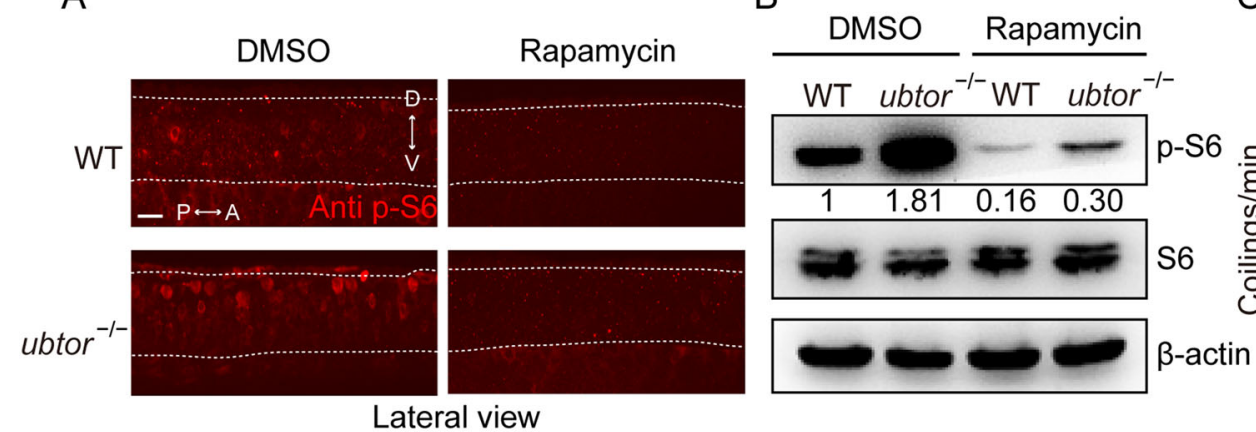

C

Lateral view

D
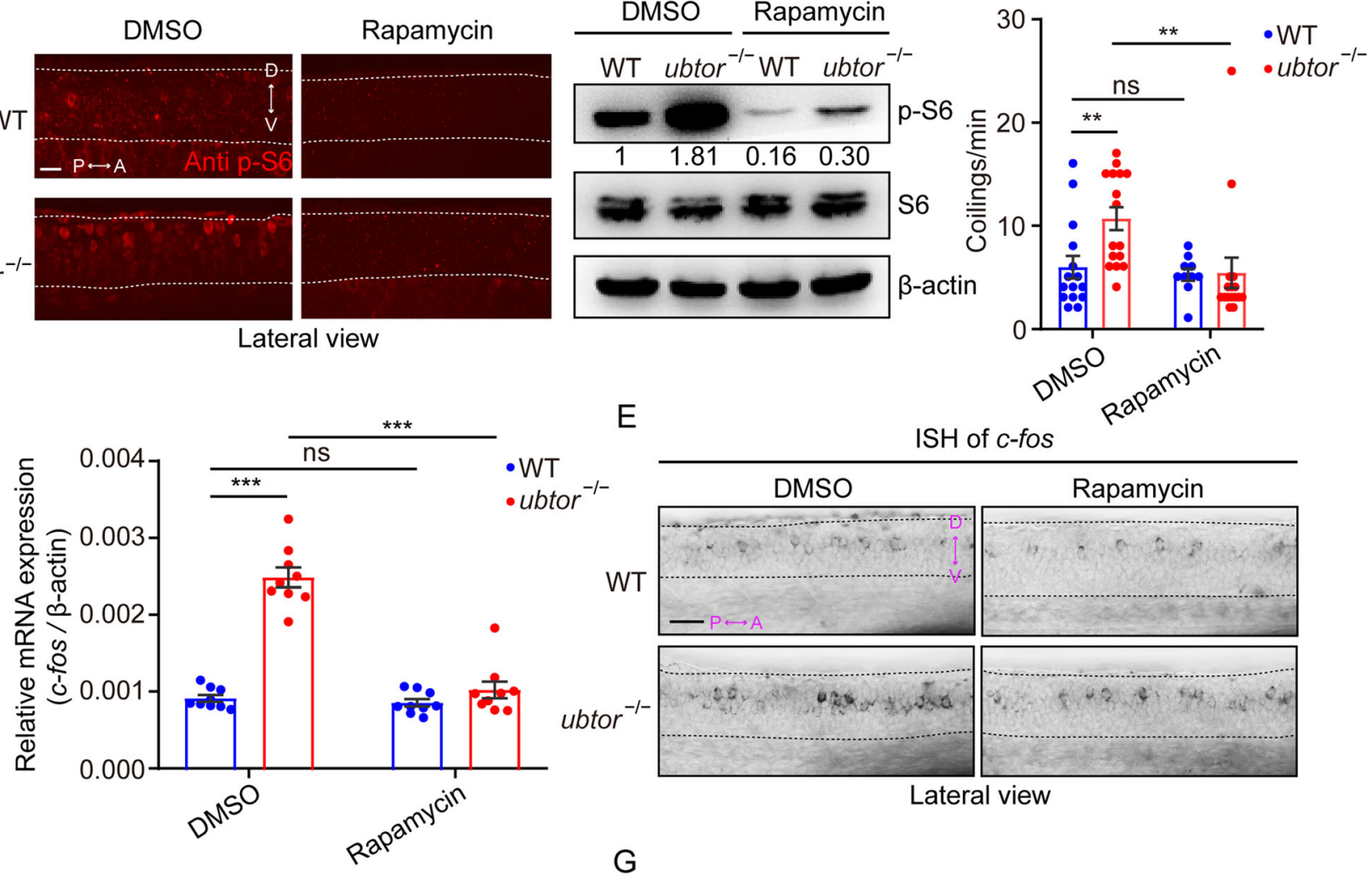

$\mathrm{F}$

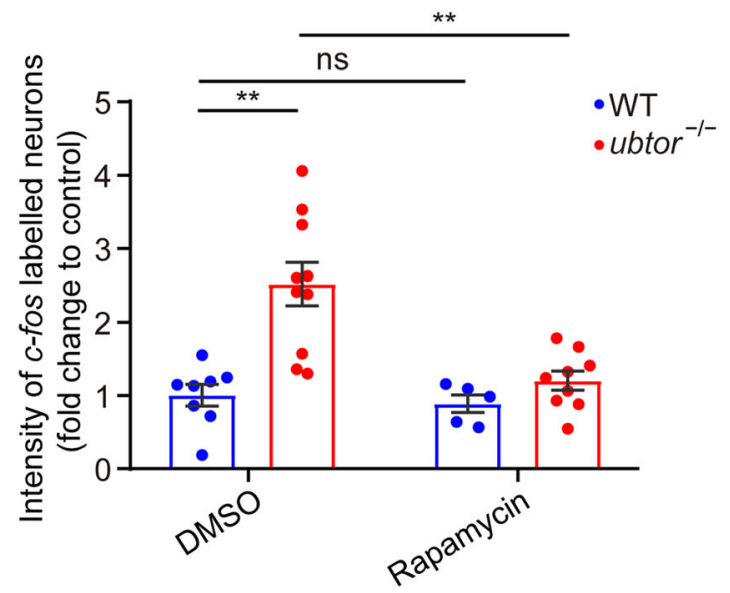

Fig. 5 Rapamycin normalizes spontaneous movements and the activity of spinal interneurons in 28-hpf ubtor mutant embryos. A p-S6 staining in the spinal cord of 28-hpf embryos treated with DMSO (solvent) or $100 \mathrm{nmol} / \mathrm{L}$ rapamycin (dashed lines, spinal somites 4-10; scale bar, $40 \mu \mathrm{m}$ ). B Immunoblotting for p-S6 and S6 protein in 28-hpf embryos treated with DMSO or $100 \mathrm{nmol} / \mathrm{L}$ rapamycin. Quantified p-S6 protein levels are indicated. C Frequency of coiling movements outside the chorion of 28-hpf embryos treated with DMSO or rapamycin (two biological repeats, DMSO: $\mathrm{N}_{\mathrm{WT}}=$ $\mathrm{N}_{\text {ubtor }}{ }^{-1-}=16, t_{29}=2.974$; rapamycin: $\mathrm{N}_{\mathrm{WT}}=\mathrm{N}_{\text {ubtor }}{ }^{-1-}=16, t_{29}=$ 0.189 ). D RT-qPCR analysis of $c$-fos mRNA levels in 28-hpf embryos treated with DMSO or rapamycin (three biological repeats, DMSO:

G
E

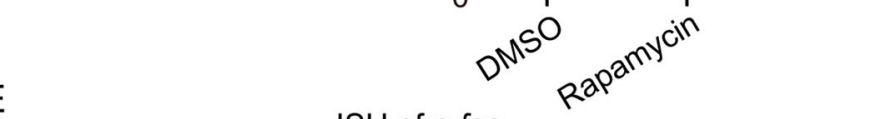

WT

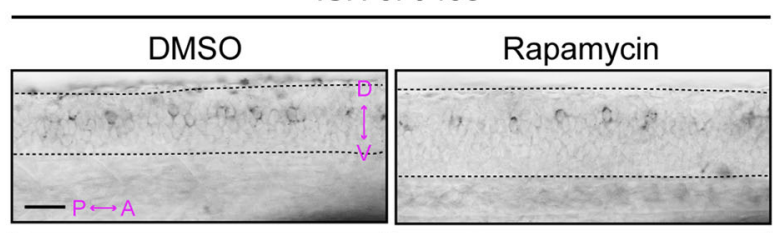

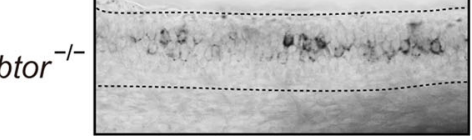

Lateral view

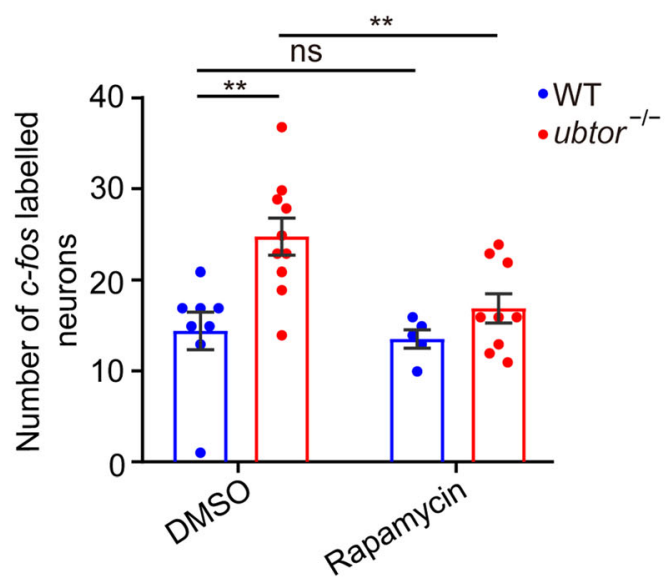

$\mathrm{N}_{\mathrm{WT}}=\mathrm{N}_{\text {ubtor }}{ }^{-/-}=75, t_{16}=11.57$; rapamycin: $\mathrm{N}_{\mathrm{WT}}=\mathrm{N}_{\text {ubtor }}{ }^{-/-}=75$, $\left.t_{16}=1.389\right)$. E Expression and distribution of $c$-fos using in situ hybridization assays in 28-hpf embryos treated with DMSO or 100 $\mathrm{nmol} / \mathrm{L}$ rapamycin (spinal somites $4-10$; scale bar, $40 \mu \mathrm{m}$ ). F, G Relative intensity (DMSO: $\mathrm{N}_{\mathrm{WT}}=8, \mathrm{~N}_{u b t o r}{ }^{-1-}=10, t_{16}=4.246$; rapamycin: $\mathrm{N}_{\mathrm{WT}}=6, \mathrm{~N}_{\text {ubtor }}{ }^{-1-}=9, t_{12}=1.586 ; \mathbf{F}$ ) and number (DMSO: $\mathrm{N}_{\mathrm{WT}}=8, \mathrm{~N}_{\text {ubtor }}{ }^{-l-}=10, t_{16}=3.520$; rapamycin: $\mathrm{N}_{\mathrm{WT}}=6$, $\left.\mathrm{N}_{\text {ubtor }}{ }^{-1-}=9, t_{12}=1.453 ; \mathbf{G}\right)$ of $c$-fos labeled spinal interneurons in 28 -hpf embryos treated with DMSO or rapamycin. $\beta$-actin served as thein ternal control. Values are represented as the mean \pm SEM in $\mathbf{C}$, D, $\mathbf{F}$, and $\mathbf{G}$. $* * P<0.01$, $* * * P<0.001$. 
A

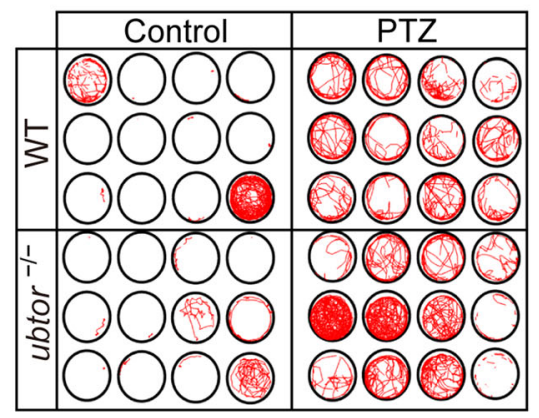

$\mathrm{B}$

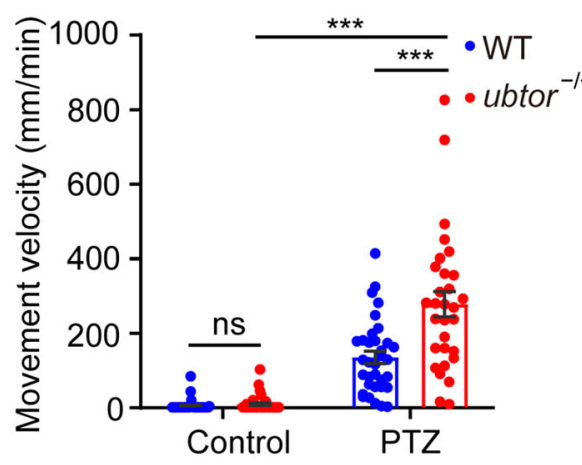

C
D

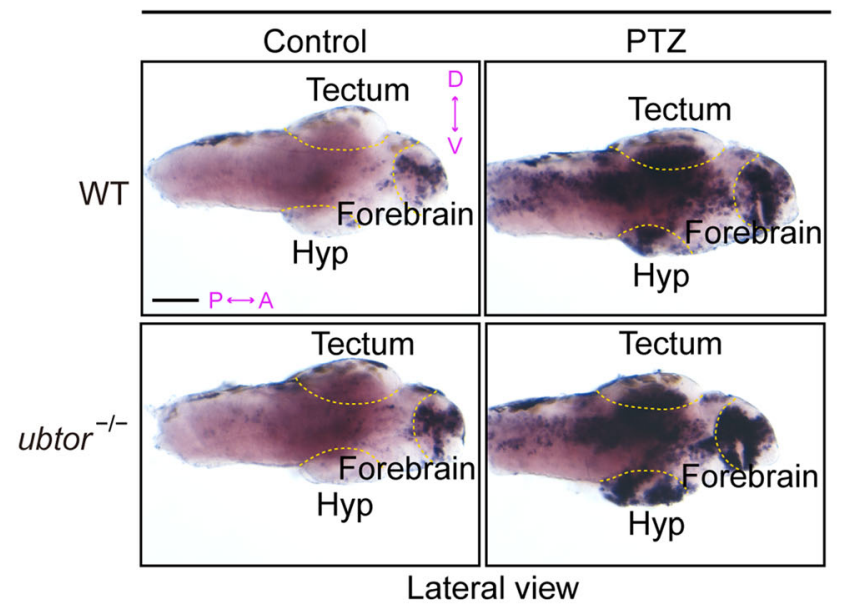

Fig. 6 ubtor mutants exhibit hypersensitivity to PTZ. A, B Locomotion tracks $\mathbf{A}$ and movement velocity $\mathbf{B}$ of 4-dpf larvae treated with water (solvent) (three biological repeats, $\mathrm{N}_{\mathrm{WT}}=\mathrm{N}_{\text {ubtor }}{ }^{-l-}=36, t_{128}=$ 0.737 ) or $3 \mathrm{mmol} / \mathrm{L}$ PTZ (three biological repeats, $\mathrm{N}_{\mathrm{WT}}=\mathrm{N}_{\text {ubtor }}{ }^{-1-}=$ $\left.36, t_{128}=4.753\right)$ in a 10 -min period. $\mathbf{C}$ Immunoblotting for $\mathrm{p}-\mathrm{S} 6$ protein in 4-dpf larvae treated with water or PTZ. Values of p-S6 protein levels are indicated. D Expression and distribution of $c$-fos

\section{Discussion}

In this study, we showed that the spontaneous movements of ubtor mutant zebrafish were increased compared with the wild-type control group, and neuronal activity in the ubtor mutant was elevated. Mechanistically, we demonstrated that mTOR signaling was increased in the ubtor mutant, and the mTORC1 inhibitor rapamycin decreased the movements and neuronal activity in the mutant. These results suggested that ubtor regulates motor hyperactivity and epilepsy-like behaviors through mTOR signaling.

UBTOR/KIAA1024/MINARI is a poorly-characterized gene. Our recent study showed that Ubtor participates in a novel negative feedback mechanism to control mTOR signaling [10]. It has also been suggested that UBTOR/ MINARl is implicated in Notch signaling and angiogenesis [13]. The in vivo function of UBTOR is largely unknown, except as a candidate gene in various cancers, and it has
E

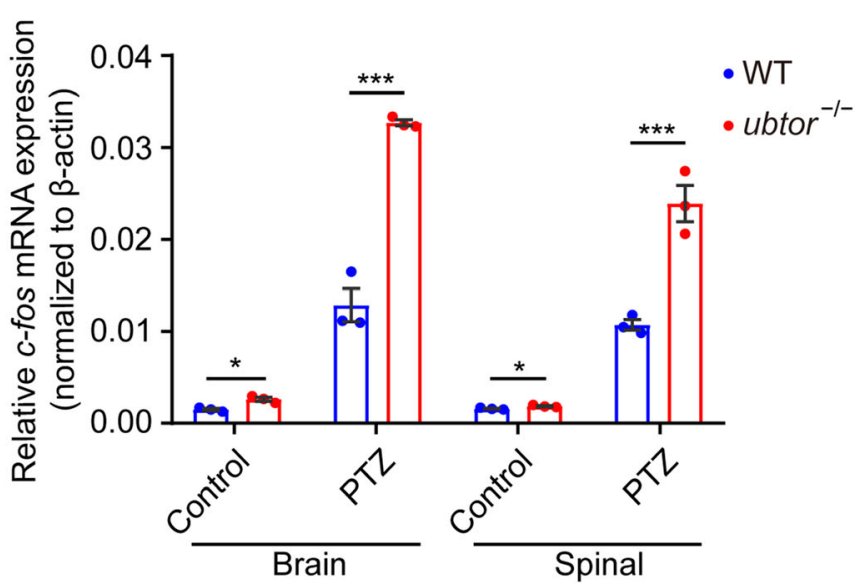

mRNA in brain tissue of 4-dpf larvae treated with solvent (water) or PTZ (scale bar, $100 \mu \mathrm{m}$ ). E RT-qPCR analysis of $c$-fos mRNA levels in brain and spinal tissues of 4-dpf larvae treated with control or PTZ (three biological repeats, control: $\mathrm{N}_{\mathrm{WT}}=\mathrm{N}_{\text {ubtor }}{ }^{-1-}=75 ; 3 \mathrm{mmol} / \mathrm{L}$ PTZ: $\left.\mathrm{N}_{\mathrm{WT}}=\mathrm{N}_{\text {ubtor }}{ }^{-1-}=75\right)$. $\beta$-actin served as the internal control. Values are represented as the mean \pm SEM in $\mathbf{B}$ and $\mathbf{E}$. $* P<0.05$, $* * * P<0.001$.

also been reported in genetic studies of neurodevelopmental disorders and intellectual disability [33]. Our results in this study showed that disruption of ubtor in zebrafish resulted in motor hyperactivity and elevated neuronal activity. These results established a role of ubtor in the nervous system in vivo. This role is also consistent with the strong ubtor expression in the central nervous system in vertebrates including fish, mice, and humans.

Loss of mTOR-negative regulators and over-activation of the mTOR signaling pathway are major causes underlying the occurrence of cortical malformations and epileptic encephalopathy. For instance, DEPDC5, a component of the GATOR1 complex and a negative regulator of mTOR, has been identified as a gene associated with focal epilepsy in humans [34]. In zebrafish, depdc5 mutant larvae show mTOR hyperactivation, epileptic discharges in the brain, and increased motor hyperactivity in response to PTZ treatment $[9,35]$. Thus, the phenotypic abnormalities 


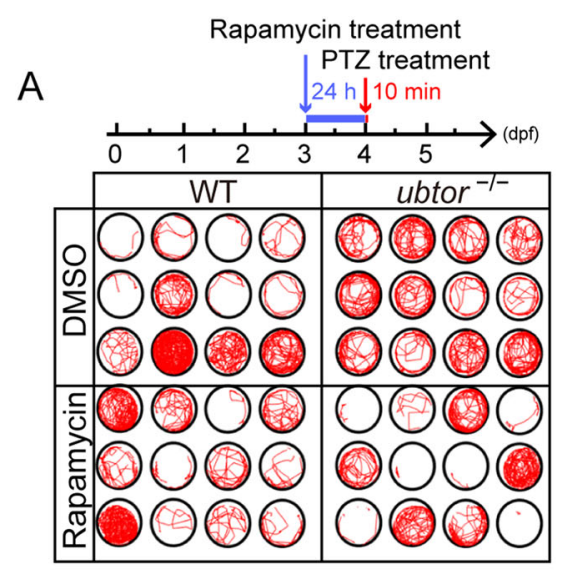

$\mathrm{D}$

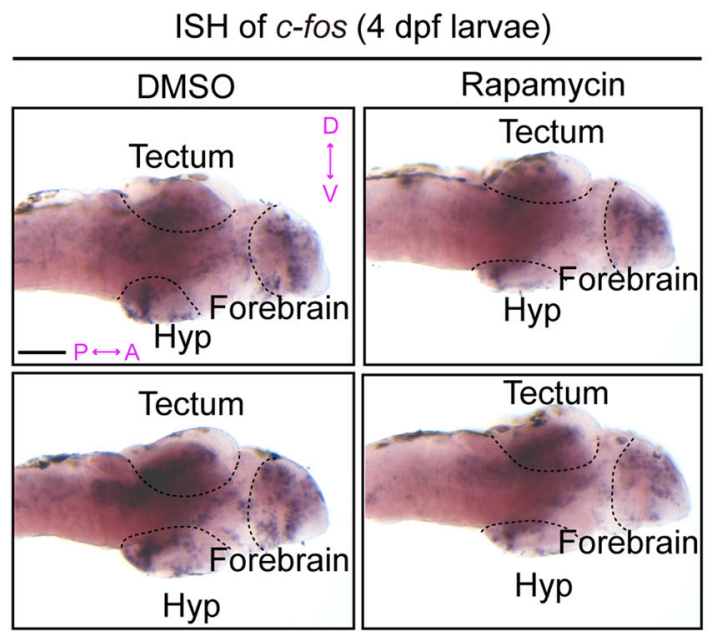

PTZ treatment

Fig. 7 Rapamycin rescues seizure susceptibility in PTZ-induced ubtor mutant larvae. A, B Locomotion tracks $\mathbf{A}$ and movement velocity $\mathbf{B}$ of 4-dpf larvae pre-treated with DMSO $\left(\mathrm{N}_{\mathrm{WT}}=\mathrm{N}_{\text {ubtor }}{ }^{-l-}=\right.$ $\left.24, t_{39}=2.987\right)$ or $2 \mu \mathrm{mol} / \mathrm{L}$ rapamycin $\left(\mathrm{N}_{\mathrm{WT}}=\mathrm{N}_{\text {ubtor }}{ }^{-1-}=24, t_{38}=\right.$ 0.365), followed by $3 \mathrm{mmol} / \mathrm{L} \mathrm{PTZ} \mathrm{(two} \mathrm{biological} \mathrm{repeats).C} \mathrm{Im-}$ munoblotting for p-S6 protein in 4-dpf larvae treated as in A. Values of p-S6 protein levels are indicated. D Expression and distribution of

found in the depdc5 mutant are similar our findings in the ubtor mutant larvae. Both the depec5 and ubtor mutation studies in zebrafish suggest that hyperactivation of mTOR signaling disrupts the balance between excitatory and inhibitory activity, which leads to abnormalities in neural functions. Our results showed that treatment with the mTORC1 inhibitor rapamycin rescued the motor hyperactivity and the elevated neuronal activity in the ubtor mutant, indicating that mTOR signaling is the major cause underlying the phenotypic abnormalities in the ubtor mutant. Treatment with rapamycin was also effective in reversing the phenotypic abnormalities found in the depdc5

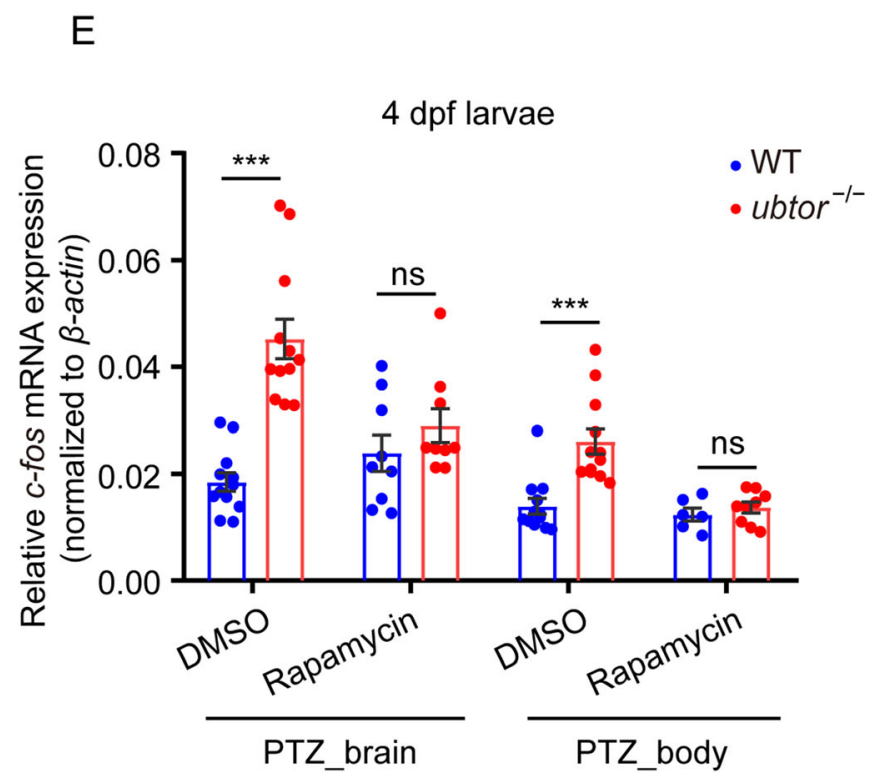

c-fos mRNA in brain tissue of 4-dpf larvae treated with rapamycin and PTZ (scale bar, $100 \mu \mathrm{m}$ ). E RT-qPCR analysis of $c$-fos mRNA levels in the brain and spinal tissues of 4-dpf larvae treated with control or rapamycin, followed by $3 \mathrm{mmol} / \mathrm{L} \mathrm{PTZ} \mathrm{(three} \mathrm{biological}$ repeats, control: $\mathrm{N}_{\mathrm{WT}}=\mathrm{N}_{\text {ubtor }}{ }^{-1-}=75$; rapamycin: $\mathrm{N}_{\mathrm{WT}}=\mathrm{N}_{\text {ubtor }}{ }^{-1-}=$ 75). $\beta$-actin served as the internal control. Values are represented as the mean \pm SEM in $\mathbf{B}$ and $\mathbf{E} . * * P<0.01, * * * P<0.001$.

mutant $[9,35]$, and in another larval mutant gene, $t s c 2$, an mTOR-negative regulator [36].

Zebrafish embryos in early development have relatively simple motor behavior and neural circuits, which can exhibit abnormal neural circuit development or activity as a result of genetic mutations. We found that the spinal interneurons of 28-hpf ubtor mutants developed normally, while $c$-fos gene expression was significantly up-regulated in spinal neurons. This result suggests that the activity of ubtor mutant spinal neurons is up-regulated. However, the specific types of spinal interneurons involved in ubtor mutants need to be identified in further studies. We further used $\mathrm{Ca}^{2+}$ imaging to examine the activity changes of 
spinal neurons at higher spatial and temporal resolution. The results showed that the neuronal activity of CiD spinal interneuron was significantly increased in ubtor mutants. Previous studies have shown that $\mathrm{CiD}$ interneurons express the excitatory neurotransmitter glutamate and function as premotor interneurons to drive the generation of motor rhythms in larvae at $52 \mathrm{hpf}$ or older [37-39]. In contrast, in vivo patch clamp electrophysiological recordings in zebrafish embryonic spinal cord neurons has shown that, at 20-24 hpf, the three primary interneurons IC (ipsilateral caudal), VeLD (ventral longitudinal descending), and CoPA are active when the embryonic trunk contracts spontaneously, while the four types of secondary interneurons, $\mathrm{CiA}$ (circumferential ascending), $\mathrm{CiD}$ (circumferential descending), CoBL (commissural bifurcating longitudinal), and CoSA, show no evidence of significant activity in the context of spontaneous behaviors [22]. This difference may be due to the fact that the axons of the secondary interneurons extend later than those of the primary interneurons and may have not yet projected to the motor neurons that drive motor behaviors [40]. The time point of our examination of spinal interneuron activity was at $28 \mathrm{hpf}$, and we saw strong and robust neuronal activity only in CiD neurons. Future experiments applying subtypespecific transgenic lines, voltage-sensitive reporters [41], and electrophysiological examination of these spinal interneurons are required to elucidate the exact roles of Ubtor in the regulation of spinal circuit activity.

Our results here showed that ubtor mutant larvae were hypersensitive to PTZ. PTZ is widely used to chemically induce seizures in mouse and zebrafish models [25, 42]. The neuropathological effects of PTZ treatment are complex; it alters both GABA-mediated inhibition and glutamate-mediated excitation [43]. Because the consequences of PTZ treatment for the ubtor mutant were highly significant, it is likely their effects were synergistic. Further examination of mTOR signaling activity and $c$-fos expression suggested that disruption of the ubtor gene enhances the sensitivity of PTZ-induced epilepsy-like behaviors by up-regulating mTORC1 pathway activity. Strikingly, rapamycin treatment restored the increased $c$-fos expression and swimming activity of ubtor mutants exposed to PTZ. Recent research has indicated that treatment with rapamycin for a period of time prior to spontaneous epilepsy or drug-induced seizures inhibits seizures or reduces their severity, which may be related to inhibition of neuronal excitability and neurotransmitter release $[30,35]$. These results further support the hypothesis that the ubtor mutation participates in the regulation of epilepsy-like behaviors through activation of the zebrafish mTORC1 pathway, thereby up-regulating neuronal activity. In future, it will be necessary to investigate how the enhancement of mTORC1 signaling caused by ubtor mutations modulated the up-regulation of neuronal activity, and to elucidate the mechanisms linked to the ubtormTOR-neuronal activity axis in the zebrafish model.

Taken together, our studies found that the ubtor gene plays a role in early spontaneous movement and epilepsy-like behaviors in zebrafish by regulating the mTOR signaling pathway and spinal interneuron activity. These salient features in the ubtor mutant zebrafish may also be used as a preclinical model for studies and screening for compounds regulating mTOR signaling and treating epilepsy.

Acknowledgements We thank Dr. K Kawakami for the GAL4FF construct and the UAS:GFP line. We thank Drs. Y Dang and Q Wang for helpful discussions. We thank $\mathrm{L} \mathrm{Gao,} \mathrm{X} \mathrm{Li,} \mathrm{and} \mathrm{C} \mathrm{Han} \mathrm{for} \mathrm{fish}$ care. This work was supported by the National Key Research and Development Program of China (2018YFA0801000), the National Natural Science Foundation of China (31371099 and 31571067), the Shanghai Municipal Science and Technology Major Project (2018SHZDZX01), ZJ Lab, and Shanghai Center for Brain Science and Brain-Inspired Technology (Shanghai, China).

Conflict of interest The authors claim that there are no conflicts of interest.

Open Access This article is licensed under a Creative Commons Attribution 4.0 International License, which permits use, sharing, adaptation, distribution and reproduction in any medium or format, as long as you give appropriate credit to the original author(s) and the source, provide a link to the Creative Commons licence, and indicate if changes were made. The images or other third party material in this article are included in the article's Creative Commons licence, unless indicated otherwise in a credit line to the material. If material is not included in the article's Creative Commons licence and your intended use is not permitted by statutory regulation or exceeds the permitted use, you will need to obtain permission directly from the copyright holder. To view a copy of this licence, visit http://creativecommons. org/licenses/by/4.0/.

\section{References}

1. Hoeffer CA, Klann E. mTOR signaling: At the crossroads of plasticity, memory and disease. Trends Neurosci 2010, 33: 67-75.

2. Zhang G, Kim S, Gu X, Yu SP, Wei L. DPP-4 inhibitor linagliptin is neuroprotective in hyperglycemic mice with stroke via the AKT/mTOR pathway and anti-apoptotic effects. Neurosci Bull 2020, 36: 407-418.

3. Crino PB (2015) Signaling in epilepsy insights from malformations of cortical development. J Matter Med 5:a022442.

4. Curatolo P, Moavero R, de Vries PJ. Neurological and neuropsychiatric aspects of tuberous sclerosis complex. Lancet Neurol 2015, 14: 733-745.

5. Crino PB, Nathanson KL, Henske EP. The tuberous sclerosis complex. N Engl J Med 2006, 355: 1345-1356.

6. Baulac S. Genetics advances in autosomal dominant focal epilepsies: Focus on DEPDC5. Prog Brain Res 2014, 213: 123-139.

7. Bar-Peled L, Chantranupong L, Cherniack AD, Chen WW, Ottina KA, Grabiner BC. A Tumor suppressor complex with GAP activity for the Rag GTPases that signal amino acid sufficiency to mTORC1. Science 2013, 340: 1100-1106.

8. Yuskaitis CJ, Jones BM, Wolfson RL, Super CE, Dhamne SC, Rotenberg A, et al. A mouse model of DEPDC5-related epilepsy: 
Neuronal loss of Depdc5 causes dysplastic and ectopic neurons, increased mTOR signaling, and seizure susceptibility. Neurobiol Dis 2018, 111: 91-101.

9. de Calbiac H, Dabacan A, Marsan E, Tostivint H, Devienne G, Ishida $\mathrm{S}$, et al. Depdc5 knockdown causes mTOR-dependent motor hyperactivity in zebrafish. Ann Clin Transl Neurol 2018, 5: 510-523.

10. Zhang HF, Zhang Q, Gao G, Wang XJ, Wang TT, Kong ZT, et al. UBTOR/KIAA1024 regulates neurite outgrowth and neoplasia through mTOR signaling. PLoS Genet 2018, 14: e1007583.

11. Giordano TJ, Thomas DG, Kuick R, Lizyness M, Misek DE, Smith AL, et al. Distinct transcriptional profiles of adrenocortical tumors uncovered by DNA microarray analysis. Am J Pathol 2003, 162: 521-531

12. Jones S, Zhang XS, Parsons DW, Lin JCH, Leary RJ, Angenendt $P$, et al. Core signaling pathways in human pancreatic cancers revealed by global genomic analyses. Science 2008, 321: 1801-1806.

13. Ho RXY, Meyer RD, Chandler KB, Ersoy E, Park M, Bondzie PA, et al. MINAR1 is a Notch2-binding protein that inhibits angiogenesis and breast cancer growth. J Mol Cell Biol 2018, 10: 195-204.

14. Asakawa K, Suster ML, Mizusawa K, Nagayoshi S, Kotani T, Urasaki A, et al. Genetic dissection of neural circuits by Tol2 transposon-mediated Gal4 gene and enhancer trapping in zebrafish. Proc Natl Acad Sci U S A 2008, 105: 1255-1260.

15. Chen F, Chen S, Liu S, Zhang C, Peng G. Effects of lorazepam and WAY-200070 in larval zebrafish light/dark choice test. Neuropharmacology 2015, 95: 226-233.

16. Pnevmatikakis EA, Giovannucci A. NoRMCorre: An online algorithm for piecewise rigid motion correction of calcium imaging data. J Neurosci Methods 2017, 291: 83-94.

17. Giovannucci A, Friedrich J, Gunn P, Kalfon J, Brown BL, Koay SA, et al. (2019) CaImAn an open source tool for scalable calcium imaging data analysis. Elife, 8:e38173.

18. Deneux T, Kaszas A, Szalay G, Katona G, Lakner T, Grinvald A, et al. Accurate spike estimation from noisy calcium signals for ultrafast three-dimensional imaging of large neuronal populations in vivo. Nat Commun 2016, 7: 12190.

19. Lewis KE. How do genes regulate simple behaviours? Understanding how different neurons in the vertebrate spinal cord are genetically specified. Philos Trans R Soc Lond B Biol Sci 2006, 361: 45-66.

20. Lewis KE, Eisen JS. From cells to circuits: Development of the zebrafish spinal cord. Prog Neurobiol 2003, 69: 419-449.

21. Drapeau P, Saint-Amant L, Buss RR, Chong M, McDearmid JR, Brustein E. Development of the locomotor network in zebrafish. Prog Neurobiol 2002, 68: 85-111.

22. Saint-Amant L, Drapeau P. Synchronization of an embryonic network of identified spinal interneurons solely by electrical coupling. Neuron 2001, 31: 1035-1046.

23. Herrera DG, Robertson HA. Activation of c-fos in the brain. Prog Neurobiol 1996, 50: 83-107.

24. Ye C, Qiu Y, Zhang F, Chen AD, Zhou H, Wang JJ, et al. Chemical stimulation of renal tissue induces sympathetic activation and a pressor response via the paraventricular nucleus in rats. Neurosci Bull 2020, 36: 143-152.

25. Baraban SC, Taylor MR, Castro PA, Baier H. Pentylenetetrazole induced changes in zebrafish behavior, neural activity and c-fos expression. Neuroscience 2005, 131: 759-768.

26. Burrows DRW, Samarut E, Liu J, Baraban SC, Richardson MP, Meyer MP, et al. Imaging epilepsy in larval zebrafish. Eur $\mathbf{J}$ Paediatr Neurol 2020, 24: 70-80.

27. Liao MJ, Kundap U, Rosch RE, Burrows DRW, Meyer MP, Ouled Amar Bencheikh B (2019) et al. Targeted knockout of GABA-A receptor gamma 2 subunit provokes transient light-induced reflex seizures in zebrafish larvae. Dis Model Mech, 12: dmm.040782.

28. Abs E, Goorden SM, Schreiber J, Overwater IE, HoogeveenWesterveld M, Bruinsma CF, et al. TORC1-dependent epilepsy caused by acute biallelic Tsc1 deletion in adult mice. Ann Neurol 2013, 74: 569-579.

29. Galanopoulou AS, Gorter JA, Cepeda C. Finding a better drug for epilepsy: The mTOR pathway as an antiepileptogenic target. Epilepsia 2012, 53: 1119-1130.

30. Marsan E, Ishida S, Schramm A, Weckhuysen S, Muraca G, Lecas S, et al. Depdc5 knockout rat: A novel model of mTORopathy. Neurobiol Dis 2016, 89: 180-189.

31. Giorgi O, Carboni G, Frau V, Orlandi M, Valentini V, Feldman A, et al. Anticonvulsant effect of felbamate in the pentylenetetrazole kindling model of epilepsy in the rat. Naunyn Schmiedebergs Arch Pharmacol 1996, 354: 173-178.

32. Siebel AM, Menezes FP, da Costa Schaefer I, Petersen BD, Bonan CD (2015) Rapamycin suppresses PTZ-induced seizures at different developmental stages of zebrafish. Pharmacol Biochem Behav, 139: 163-168.

33. Coste de Bagneaux P, von Elsner L, Bierhals T, Campiglio M, Johannsen J, Obermair GJ et al. (2020) A homozygous missense variant in $\mathrm{CACNB}_{4}$ encoding the auxiliary calcium channel beta4 subunit causes a severe neurodevelopmental disorder and impairs channel and non-channel functions. PLoS Genet, 16: 1008625.

34. Anderson MP. DEPDC5 takes a second hit in familial focal epilepsy. J Clin Invest 2018, 128: 2194-2196.

35. Swaminathan A, Hassan-Abdi R, Renault S, Siekierska A, Riché $\mathrm{R}$, Liao $\mathrm{M}$, et al. Non-canonical mTOR-independent role of DEPDC5 in regulating GABAergic network development. Curr Biol 2018, 28: 1924-1937.e5.

36. Scheldeman C, Mills JD, Siekierska A, Serra I, Copmans D, Iyer $\mathrm{AM}$, et al. mTOR-related neuropathology in mutant tsc2 zebrafish: Phenotypic, transcriptomic and pharmacological analysis. Neurobiol Dis 2017, 108: 225-237.

37. Kimura Y, Okamura Y, Higashijima SI. Alx, a zebrafish homolog of Chx10, marks ipsilateral descending excitatory interneurons that participate in the regulation of spinal locomotor circuits. J Neurosci 2006, 26: 5684-5697.

38. McLean DL, Fan JY, Higashijima SI, Hale ME, Fetcho JR. A topographic map of recruitment in spinal cord. Nature 2007, 446: 71-75.

39. Eklöf-Ljunggren E, Haupt S, Ausborn J, Dehnisch I, Uhlén P, Higashijima $\mathrm{S}$, et al. Origin of excitation underlying locomotion in the spinal circuit of zebrafish. Proc Natl Acad Sci U S A 2012, 109: 5511-5516.

40. Bernhardt RR, Chitnis AB, Lindamer L, Kuwada JY. Identification of spinal neurons in the embryonic and larval zebrafish. J Comp Neurol 1990, 302: 603-616.

41. Villette V, Chavarha M, Dimov IK, Bradley J, Pradhan L, Mathieu B, et al. Ultrafast two-photon imaging of a high-gain voltage indicator in awake behaving mice. Cell 2019, 179: 1590-1608.e23.

42. Löscher W. Critical review of current animal models of seizures and epilepsy used in the discovery and development of new antiepileptic drugs. Seizure 2011, 20: 359-368.

43. Samokhina E, Samokhin A. Neuropathological profile of the pentylenetetrazol (PTZ) kindling model. Int J Neurosci 2018, 128: $1086-1096$. 